\title{
Double miss by ultrasound of a cornual ectopic pregnancy
}

\author{
Daniel Myran (Meds 2014) \\ Faculty Reviewer: Dr Meagan O'Brien, MD, FRCSC
}

\begin{abstract}
Cornual ectopic pregnancy is a rare subtype of ectopic pregnancy that is associated with later presentation and higher likelihood of mortality. Despite advances in sonography cornual ectopic pregnancies remain a challenging diagnosis. We present a case of a left cornual pregnancy that was initially interpreted as a live intrauterine pregnancy by sonographic examination and found during repeat assessment to be a cornual pregnancy.
\end{abstract}

\section{INTRODUCTION}

Ectopic pregnancy, a pregnancy occurring outside the uterus, is one of the most common causes of first trimester bleeding and the most common life-threatening emergency in early pregnancy. ${ }^{1}$ Currently, $2 \%$ of pregnancies result in an ectopic pregnancy, of which $95 \%$ occur in the body of fallopian tubes. ${ }^{2}$ Interstitial ectopic pregnancies (cornual pregnancies) are a rare subtype of ectopic pregnancy, resulting when implantation occurs in the most proximal part of the fallopian tube. Cornual pregnancies occur in approximately 2 to 4 percent of ectopic pregnancies or 1 in every 2500 to 5000 pregnancies. ${ }^{3}$ Cornual pregnancies are a life-threatening condition with an associated maternal mortality rate of 2 to $2.5 \%$ compared to the overall $0.14 \%$ mortality rate for ectopic pregnancy. ${ }^{3,4}$

We report here a case of a cornual pregnancy that was twice interpreted by sonographic examination as a normal intrauterine gestation before diagnosis and treatment with an open resection.

\section{CASE PRESENTATION}

A 33-year-old woman, gravida 6, para 3 with two previous miscarriages, presented to the emergency department with a one-week history of left lower quadrant pain that had worsened over the past 24 hours. In addition, the patient noted vaginal spotting occurring in the past 24-hour period. Her menstrual periods had been regular, lasting 5 days with a 28 -day cycle. At the time of presentation she was at 10 weeks gestation age by last menstrual period. The patient was otherwise healthy with no previous pelvic surgeries, no known history of sexually transmitted infections, and no history of assisted fertilization.

Vitals of the patient at triage were as follows: blood pressure $100 / 73$, temperature $36.2^{\circ} \mathrm{C}$, heart rate 81 , and respiratory rate 16 . Physical exam showed a stable, well-appearing 33 year old with scant vaginal bleeding. A solid tender mass in the left lower quadrant was palpated.

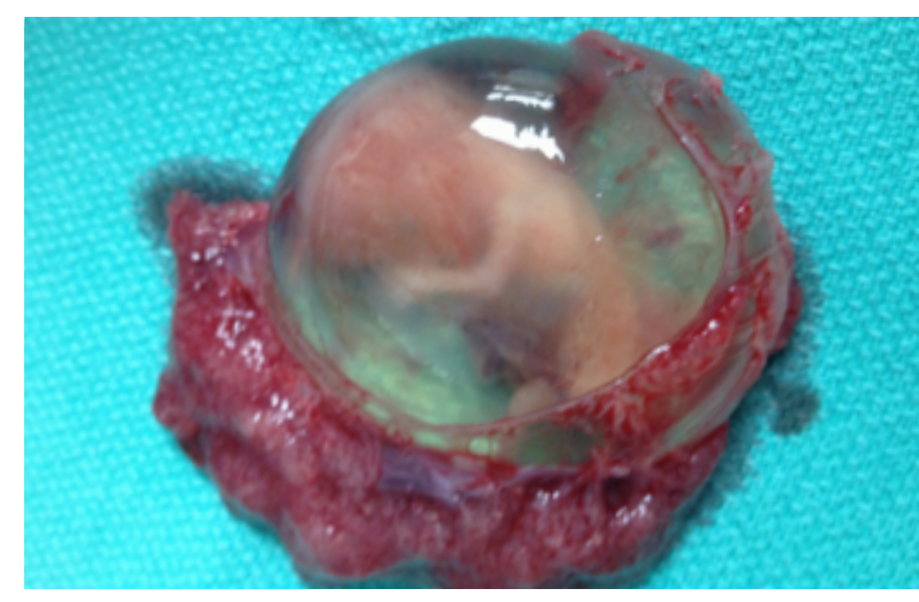

Image 1: Intact cornual ectopic pregnancy removed from patient.

\section{INVESTIGATIONS}

A transabdominal ultrasound, performed one week prior at an outside institution for routine dating, was interpreted as showing a viable intrauterine pregnancy at 10 weeks gestation. A bedside transabdominal ultrasound performed by the emergency physician on call at the time of presentation was interpreted as showing a viable intrauterine pregnancy. Given the clinical picture, formal repeat ultrasound in the radiology department was conducted on the following day. Ultrasound findings were "an empty uterus and a gestational sac and embryo with motion and cardiac activity located in the left adnexal region with a moderately prominent endometrial canal.”

The patient was taken for emergency laparotomy. A nonruptured amniotic sac measuring $5 \times 5-\mathrm{cm}$ with a fetus inside (pictured below) was visualized and removed. A left salpingectomy and repair of uterine cornua were performed and the incision was closed. The patient had an uneventful postoperative course and was discharged from hospital.

\section{DISCUSSION}

\section{ANATOMY}

The anatomy of a cornual pregnancy is responsible for both its late presentation and increased mortality. Cornual pregnancies are located in the proximal portion of the fallopian tube and surrounded by the muscle of the myometrium. The surrounding myometrium allows for greater distensibility before rupture and results in presentation at later stages than in a typical ectopic pregnancy, often as late as 12 to 16 weeks. ${ }^{4}$ The cornual implantation site is also located between the uterine and ovarian arteries. Consequently, expansion and rupture of the implantation leads to increased risk of life-threatening hemorrhage. ${ }^{5}$ 


\section{CASE REPORT}

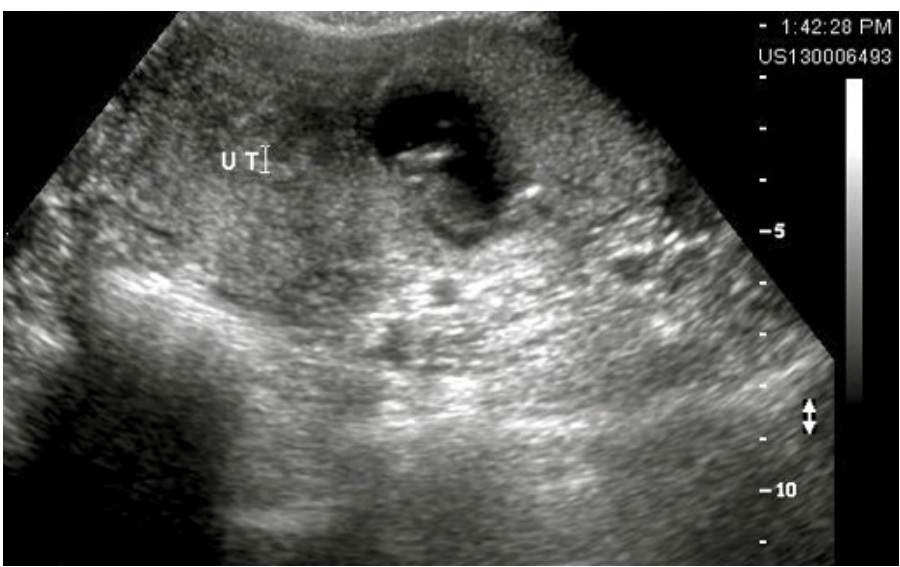

Image 2. Ultrasound image from patient left sagittal view of uterus showing gestational sac on cornua.

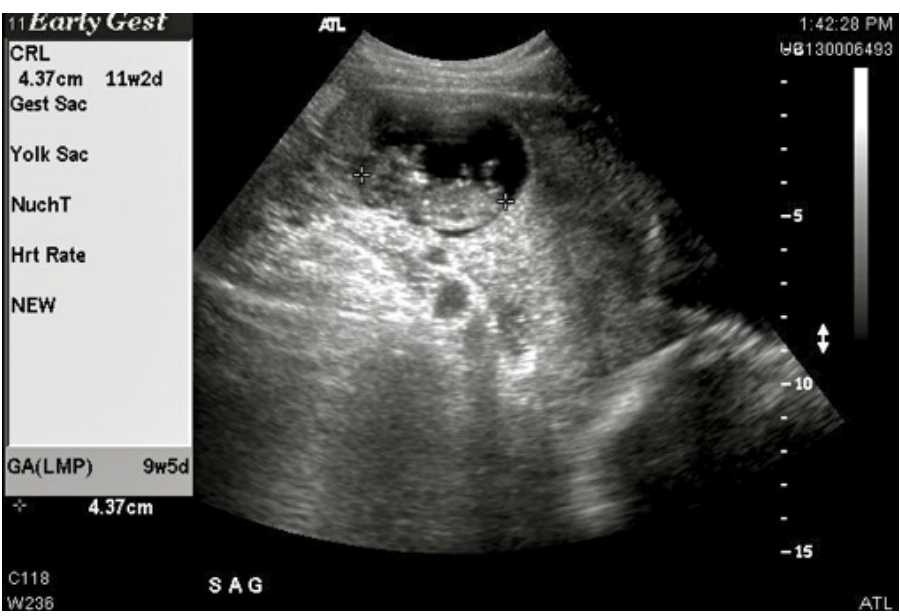

Image 3. Ultrasound image from patient showing the crown to rump length (CRL) for gestation. CRL is used to date early pregnancies; it is highly reproducible and up to 12 weeks is the best way of predicting gestational age. Our patient's CRL showed an 11-week 2-day gestation.

\section{RISK FACTORS}

Risk factors for cornual pregnancy are the same as those for ectopic pregnancy. These include pelvic inflammatory disease, previous pelvic surgeries including ruptured appendix, uterine abnormalities, and artificial fertilization. ${ }^{5}$

\section{DIAGNOSIS}

Of any pregnant patient presenting to the emergency room with first trimester bleeding or pain, 6 to $16 \%$ will have an ectopic pregnancy. ${ }^{5}$ Even with a high index of suspicion and advances in sonography, including transvaginal sonography and serum beta hCG, cornual pregnancy remains the most difficult type of ectopic pregnancy to diagnose due to the low sensitivity and specificity of symptoms and imaging. ${ }^{3}$ The classic diagnostic triad of ectopic pregnancy - abdominal pain, amenorrhea, and vaginal bleeding occurs in less than $40 \%$ of patients with cornual pregnancy. ${ }^{5}$ This triad is also present in ectopic pregnancy, threatened, and incomplete abortions. Cornual pregnancies show fewer early symptoms due to the increased distensibility of the uterine wall. ${ }^{6}$ The site of implantation also makes the pregnancy difficult to differentiate from an intrauterine pregnancy on ultrasound. ${ }^{4,7}$ As was seen in our case, transabdominal ultrasound can miss the diagnosis as the pregnancy may appear to be in an intrauterine location.

\section{TREATMENT}

With the development and increasing availability of transvaginal ultrasound, the diagnosis of cornual pregnancy is more frequently being made before rupture.

Traditionally cornual ectopic pregnancies were treated with hysterectomy. The current gold standard for a cornual pregnancy is a diagnostic laparotomy followed by a cornual wedge resection. Recent surgical advances have demonstrated that operative laparoscopy is a possible alternative in a stable patient with no suspicion of rupture. ${ }^{8}$

Conservative methods of treating cornual ectopic pregnancies include systemic administration of methotrexate and laprascopy-assisted or ultrasound-guided injection of methotrexate into the gestational sac. ${ }^{1}$ Conservative methods have been demonstrated to have an up to $65 \%$ success rate with systemic treatment. ${ }^{9}$ Local injections appear to be more effective but are operator dependent. ${ }^{9}$ All conservative methotrexate-based therapy is dependent on a stable patient and early diagnosis.

\section{CONCLUSION}

Cornual pregnancy remains a rare but significant diagnostic challenge. With increasing use of assisted reproductive technologies its incidence is expected to rise. A high index of suspicion should be maintained in any patient presenting with heavy bleeding during the first trimester. Repeat sonographic assessment is essential even in light of a "normal” intrauterine pregnancy if a patient's symptoms are consistent with ectopic pregnancy. Diagnosis before rupture is essential to prevent mortality and potential loss of fertility.

\section{REFERENCES}

1. Murray H, Baakdah H, Bardell T, Tulandi T. Diagnosis and treatment of ectopic pregnancy. CMAJ. 2005 Oct;173(8):905-12.

2. Goldner TE, Lawson HW, Xia Z, Atrash HK. Surveillance for ectopic pregnancy - United States, 1970-1989. MMWR CDC Surveill Summ. 1993 Dec;42(6):73-85.

3. Radwan F, Steel M. Review Management of cornual (interstitial) pregnancy. Obstet \& Gynaecol. 2007 Jan;9(4):249-55.

4. Wright CT. Sonographic Evaluation of Interstitial (Cornual) Ectopic Pregnancy. J Diagn Med Sonogr. 2008 Oct;24:374-9.

5. Kun W, Tung W. On the Look out for a rarity - interstitial / cornual pregnancy. Eur J of Emerg Med. 2001;8(2):147-50.

6. Molinaro TA, Barnhart KT. Ectopic Pregnancies in Unusual locations. Semin Reprod Med. 2007;25(2):123-30.

7. Auslender R, Arodi J, Pascal B, Abramovici H. Interstitial pregnancy: early diagnosis by ultrasonography. Am J Obstet Gynecol. 1983 Jul;146(6):717-8.

8. Walid MS, Heaton RL. Diagnosis and laparoscopic treatment of cornual ectopic pregnancy. Ger Med Sci. 2010 Jul;8:1-4.

9. Lau S, Tulandi T. Conservative medical and surgical management of interstitial ectopic pregnancy. Fertil Steril. 1999 Aug;72(2):207-15.

10. Bowman, ES. Nonepileptic seizures: psychiatric framework, treatment, and outcome. Neurology.1999;53(5 Suppl 2):S84-8. 